\title{
CT Colonography: Role of a second reader CAD paradigm in the initial training of radiologists
}

\author{
Emanuele Neri ${ }^{\mathrm{a}, *}$, Lorenzo Faggioni ${ }^{\mathrm{a}}$, Daniele Regge ${ }^{\mathrm{b}}$, Paola Vagli ${ }^{\mathrm{a}}$, Francesca Turini $^{\mathrm{a}}$, \\ Francesca Cerri ${ }^{a}$, Eugenia Picano ${ }^{a}$, Sabina Giusti $^{a}$, Carlo Bartolozzi ${ }^{a}$ \\ a Diagnostic and Interventional Radiology, University of Pisa, Italy \\ ${ }^{\mathrm{b}}$ Institute for Cancer Research and Treatment, Candiolo, Italy
}

\section{A R T I C L E I N F O}

\section{Article history:}

Received 15 January 2010

Received in revised form 19 July 2010

Accepted 19 July 2010

\section{Keywords:}

CT colonography

Virtual colonoscopy

Computed tomography

Colorectal polyps

Computer aided diagnosis

\begin{abstract}
A B S T R A C T
Purpose: To evaluate the influence of CAD for the evaluation of CT colonography (CTC) datasets by inexperienced readers during the attendance of a dedicated hands-on training course.

Method and materials: Twenty-seven radiologists inexperienced in CTC (11 with no CTC training at all, 16 having previously reviewed no more than 10 CTC cases overall) attended a hands-on training course based on direct teaching on fifteen workstations (four Advantage Windows 4.4 with Colon VCAR software, GE; six CADCOLON, Im3D; five ColonScreen (Toshiba/Voxar) with ColonCAD ${ }^{\mathrm{TM}}$ API, Medicsight). During the course, readers were instructed to analyze 26 CTC cases including 38 colonic lesions obtained through low-dose MDCT acquisitions, consisting of 12 polyps sized less than $6 \mathrm{~mm}, 9$ polyps sized between 6 and $10 \mathrm{~mm}, 12$ polyps sized between $11 \mathrm{~mm}$ and $30 \mathrm{~mm}$, and 5 colonic masses sized $>3 \mathrm{~cm}$. CTC images were reviewed by each reader both in 2D and 3D mode, respectively by direct evaluation of native axial images and MPR reconstructions, and virtual endoscopy or dissected views. Each reader had 15 min time for assessing each dataset without CAD, after which results were compared with those provided by CAD software. Global rater sensitivity for each lesion size before and after CAD usage was compared by means of two-tailed Student's $t$ test, while sensitivity of each single reader before and after CAD usage was assessed with the McNemar test.

Results: For lesions sized $<6 \mathrm{~mm}$, global rater sensitivity was $0.1852 \pm 0.1656$ (mean $\pm \mathrm{SD}$ ) before CADassisted reading and $0.2345 \pm 0.1761$ after $\operatorname{CAD}(p=0.0018)$. For lesions sized $6-9 \mathrm{~mm}$, sensitivity was $0.2870 \pm 0.1016$ before CAD-assisted reading and $0.3117 \pm 0.1099$ after $\operatorname{CAD}(p=0.0027)$. For lesions sized $10-30 \mathrm{~mm}$, sensitivity was $0.5308 \pm 0.2120$ before CAD-assisted reading and $0.5637 \pm 0.2133$ after CAD $(p=0.0086)$, while for lesions sized $>30 \mathrm{~mm}$, sensitivity before CAD-assisted reading was $0.3556 \pm 0.3105$ and did not change after CAD usage $(p=1)$. Sensitivity of each single rater did not significantly differ before and after CAD for any lesion size category (McNemar test, $p>0.05$ ). Specificity was not significantly different before and after CAD for any lesion size ( $>96 \%$ for all size categories).

Conclusion: CAD usage led to increased overall sensitivity of inexperienced readers for all polyps sizes, except for lesions $>30 \mathrm{~mm}$, but sensitivity of individual raters was not significantly higher compared with CAD-unassisted reading.
\end{abstract}

(C) 2010 Elsevier Ireland Ltd. All rights reserved.

\section{Introduction}

As computed tomography colonography (CTC) continues to evolve and improve, its use is shifting from highly specialized academic centers to community hospitals and nonacademic radiology practices [1-3]. Thus, many radiologists are experiencing pressure

\footnotetext{
* Corresponding author at: Diagnostic and Interventional Radiology, Department of Oncology Transplants \& Advanced Technologies in Medicine, Faculty of Medicine and Surgery, University of Pisa, Radiodiagnostica 1 Universitaria, Ospedale Cisanello. Via Paradisa 2, 56100 Pisa, Italy.

E-mail address: eneri@sirm.org (E. Neri).
}

from clinical colleagues to offer CTC as part of the routine services provided in their practices, and hands-on courses at reading workstations are increasingly being encouraged as the most suitable method for training and certify CTC readers [4].

Manual reading of CTC images is increasingly prone to errors due to the high number of images to be analyzed, which may lead to reader's fatigue [5]. Moreover, image interpretation is subjected to reader's bias (as high incidence of perceptual errors and fatigue) and no systematic method has been designed so far for lesion visualization (2D or 3D) [6].

In the attempt at overcoming the limitations of softwareunassisted reading of CTC datasets, several works have addressed the issue of evaluating the diagnostic performance of CAD (Com- 
Table 1

Mean per-polyp sensitivity and false positive rates of CAD systems.

\begin{tabular}{|c|c|c|c|}
\hline CAD & CAD \#1 & CAD \#2 & CAD \#3 \\
\hline Mean sensitivity $(<6 \mathrm{~mm})$ & $0.8532 \pm 0.2610$ & $0.8124 \pm 0.1917$ & $0.8693 \pm 0.3452$ \\
\hline Mean FP rate $(<6 \mathrm{~mm})$ & $0.1852 \pm 0.4833$ & $0.1683 \pm 0.3754$ & $0.2154 \pm 0.3255$ \\
\hline Mean sensitivity (6-9 mm) & $0.8734 \pm 0.1622$ & $0.8593 \pm 0.2403$ & $0.8195 \pm 0.2221$ \\
\hline Mean FP rate (6-9 mm) & $0.2504 \pm 0.3389$ & $0.3088 \pm 0.3674$ & $0.2455 \pm 0.3771$ \\
\hline Mean sensitivity (10-30 mm) & $0.9290 \pm 0.3703$ & $0.9088 \pm 0.3831$ & $0.8878 \pm 0.3068$ \\
\hline Mean FP rate (10-30 mm) & $0.3135 \pm 0.2754$ & $0.2710 \pm 0.3276$ & $0.2643 \pm 0.3411$ \\
\hline Mean sensitivity (>30 mm) & $0.9843 \pm 0.2938$ & $0.9567 \pm 0.4245$ & $0.9901 \pm 0.4955$ \\
\hline Mean FP rate $(>30 \mathrm{~mm})$ & $0.1852 \pm 0.3958$ & $0.1755 \pm 0.2398$ & $0.2319 \pm 0.3403$ \\
\hline
\end{tabular}

puter Aided Diagnosis) systems for CTC [7-12]. Most of the studies focus on the impact of CAD as second reader in experienced readers, however it is of interest to evaluate the impact of CAD systems on the diagnostic performance of readers without dedicated CTC experience, as could be those working in nonacademic centers, involved in reading large amount of CTC generated by a screening program. In this direction, the aim of our work was to evaluate the influence of CAD on the diagnostic performance of radiologists with no previous CTC training who have attended a residential hands-on CTC course.

\section{Materials and methods}

\subsection{Training of radiologists and case selection}

Twenty-seven radiologists with no previous CTC experience attended a three-day hands-on training course held at our Depart- ment, based on direct teaching on workstations. All of them had received no prior CTC training; 16 reported to have occasionally reviewed (not reported) a CTC exam in a clinical setting (on average 4 cases; minimum 2 and maximum 10 ) without the supervision of an expert; anyway, for the purpose of the study, at the beginning of the course all readers declared to the leading author (EN) that they have not received training in CTC before, and they had no experience in CTC reading.

The course was held over three consecutive days with $5 \mathrm{~h}$ of preliminary training to interpretation of CTC images and usage of workstations and CAD software. During the preliminary training the novices were simply trained on the use of the software tools, i.e. prone and supine images synchronization and scrolling, fly-through with endoscopic simulation, generation of a virtual dissected view, usage of measurement and reporting tools, and starting a CAD session in a second reader paradigm. All novices were trained on how to use the software with a set of three negative and (a)

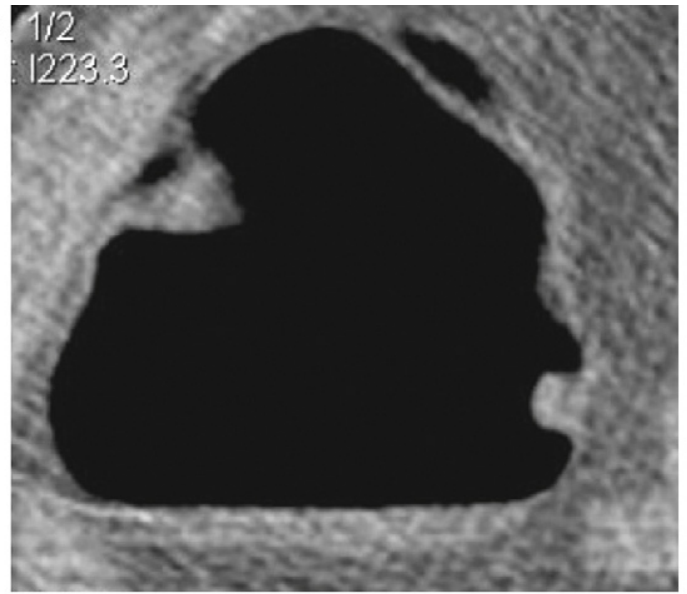

(b)

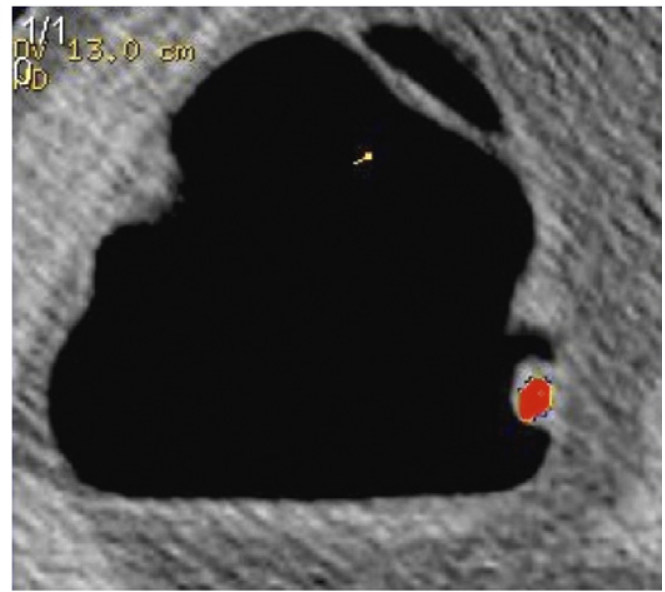

(c)

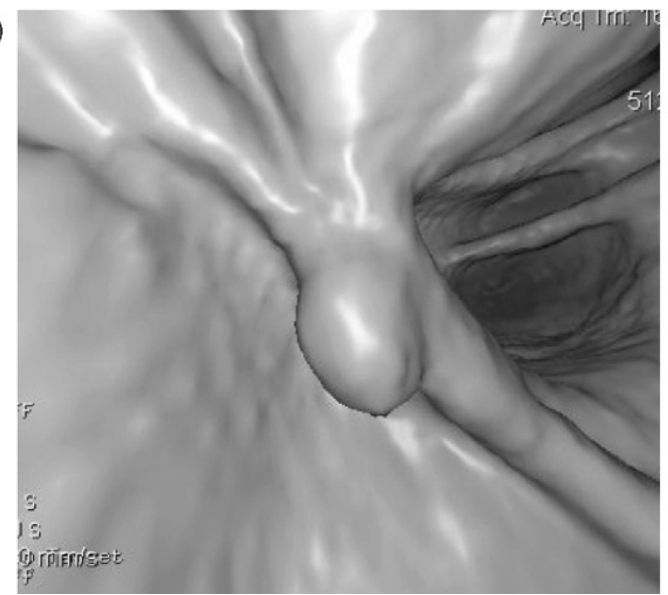

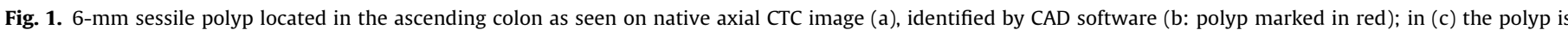
visualized in virtual endoscopy mode. The CAD-proposed candidate polyp was rejected by 7 readers and accepted by 11 readers; CAD confirmed diagnosis of 9 readers. 
(a)

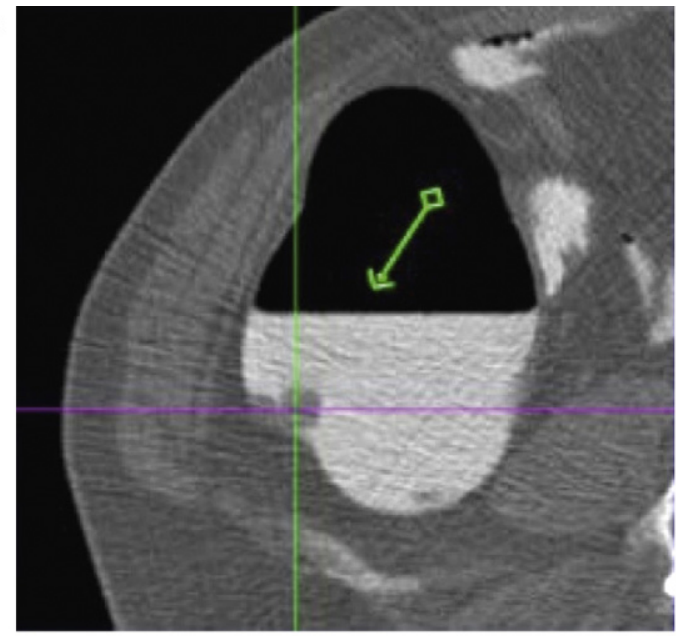

(b)

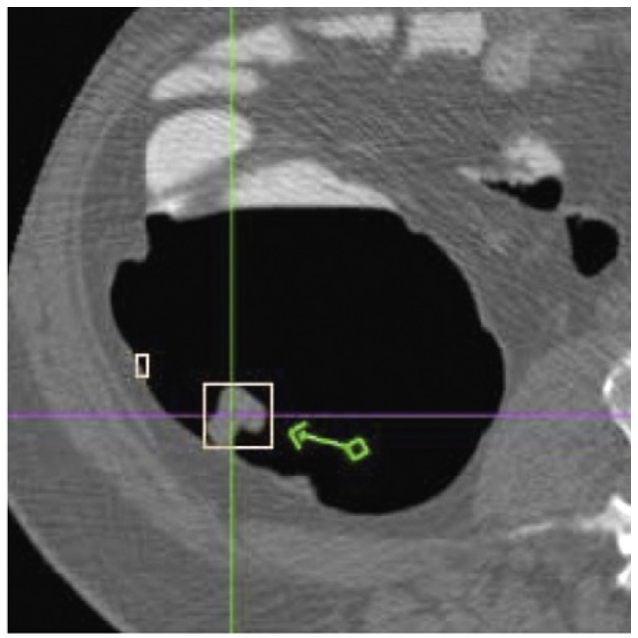

(c)

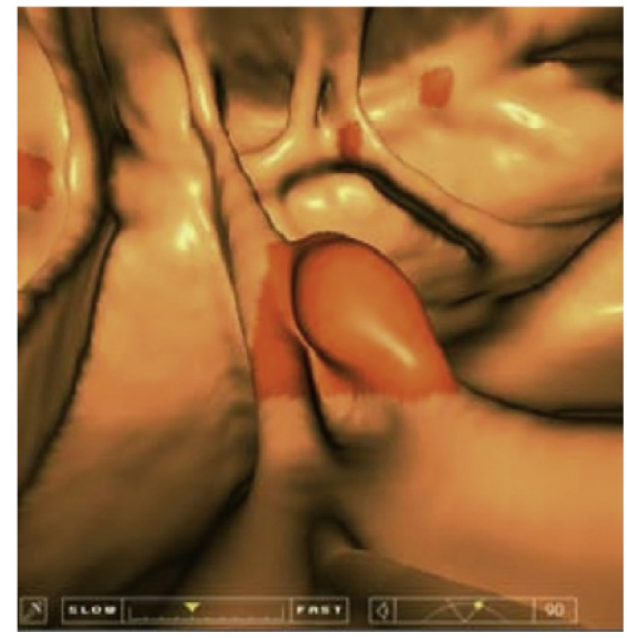

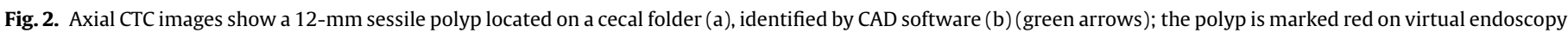
view (c). The CAD-proposed candidate polyp was rejected by 8 readers and accepted by 13 readers; CAD confirmed diagnosis of 6 readers.

three positive cases (with lesions of mixed size), in which diagnosis was given prior to training. The use of positive cases was aimed at training the novices on the use of measurement and reporting tools, as well as the recognition of the CAD markers.

Subsequently, 26 cases (excluding those already reviewed in the preliminary training) with 38 colonic lesions obtained through lowdose MDCT acquisitions were presented, consisting of 12 polyps sized less than $6 \mathrm{~mm}, 9$ polyps sized between 6 and $10 \mathrm{~mm}, 12$ polyps sized between $11 \mathrm{~mm}$ and $30 \mathrm{~mm}$, and 5 colonic masses (>3 cm size).

Institutional review board approval was waived since all cases were retrospectively selected among previous CTC studies with a colonscopic correlation, performed in our Department. All patients gave written informed consent for CTC.

\subsection{Data acquisition}

CTC datasets were obtained in 19 cases after a cathartic preparation based on oral introduction of $4 \times 37.9 \mathrm{~g}$ of polyethilenglycole (PEG) diluted in 3-4 L of water $24 \mathrm{~h}$ before CTC. In seven cases a fluid tagging preparation was employed, consisting of $6 \times 30 \mathrm{~mL}$ diatrizoate dimeglumine (Gastrografin ${ }^{\mathrm{TM}}$, Bracco Diagnostics, Milan, Italy) administered orally two days before CTC, diluted into $100 \mathrm{~mL}$ of water after every meal (six doses for a total of $180 \mathrm{~mL}$ of diatrizoate dimeglumine diluted into $600 \mathrm{~mL}$ of water). In both cases, a residue-free diet was prescribed starting 3 days before CTC.
All data were acquired on a 64-row MDCT scanner (LightSpeed VCT, GE Medical Systems, Milwaukee, WI) in the supine and prone position with the following parameters: tube voltage $120 \mathrm{kV}$, tube current 50-80 mA depending on patient size, rotation time $500 \mathrm{~ms}$, beam pitch $1.375: 1$, detector configuration $64 \times 0.625 \mathrm{~mm}$, reconstructed slice thickness $1.25 \mathrm{~mm}$, reconstruction increment $1 \mathrm{~mm}$, standard reconstruction kernel.

\subsection{Image reading}

All CTC datasets were selected and reviewed by an experienced reader (EN) according to the following criteria: radiological findings had been confirmed by means of optical colonoscopy, and data should also have optimal colonic cleansing, distention, and tagging, and to be polyp-enriched.

CT images were exported in DICOM format to 15 workstations equipped with CAD software for evaluation of CTC datasets working in a second reader paradigm (four Advantage Windows 4.4 with Colon VCAR software, GE Medical Systems [CAD \#1]; six CADCOLON, Im3D, Turin, Italy [CAD \#2]; five ColonScreen (Toshiba/Voxar) with ColonCAD ${ }^{\mathrm{TM}}$ API, Medicsight PLC, London, UK [CAD \#3]).

All cases were pre-processed by each CAD. Mean per-polyp sensitivity and false positive rate was calculated for each CAD (Table 1).

Each workstation was set to run with its own standard factory CAD settings and was assigned to one or two readers: in this latter case, the second reader assessed CTC images after the first one and 
(a)

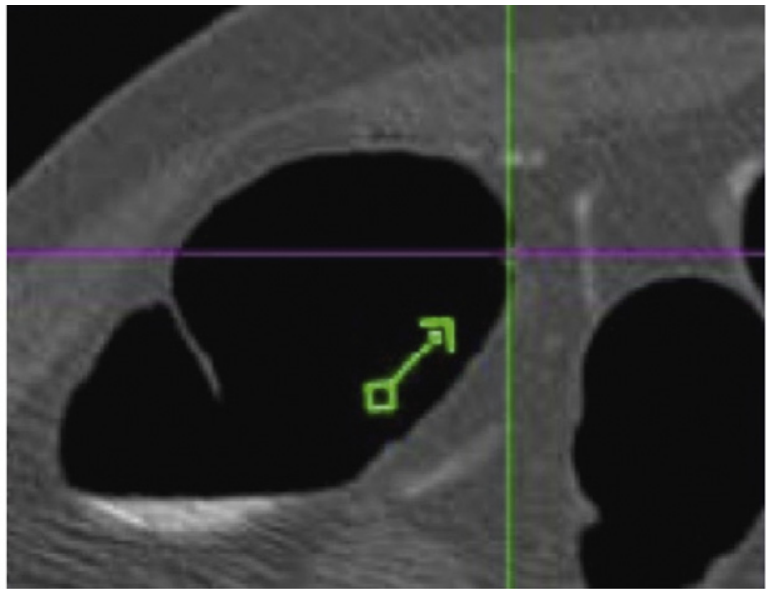

(b)

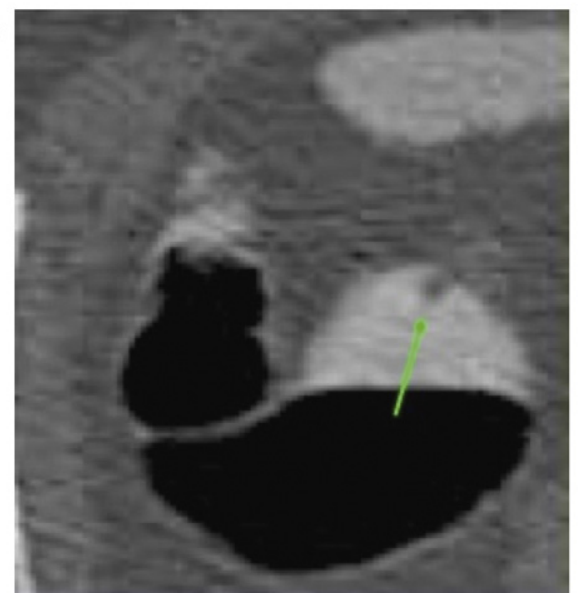

(c)

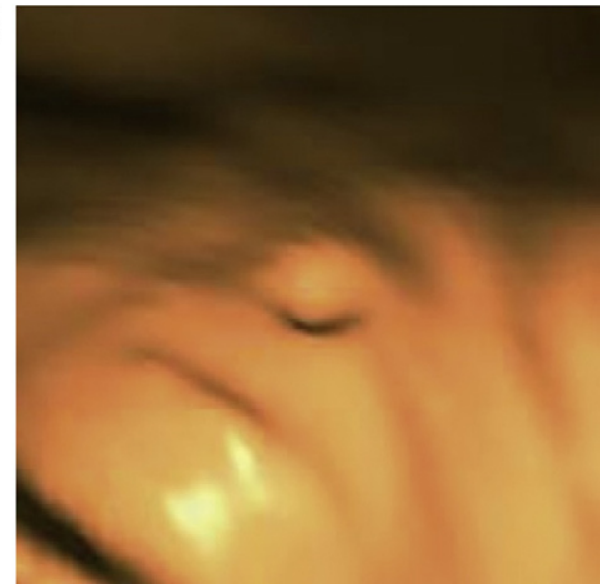

Fig. 3. Axial CTC images show a 5-mm sessile polyp (a, b: green arrows), which was not identified by both readers and CAD software (green arrows); (c) colonic wall visualized with virtual endoscopy algorithm.

in blind to him. All raters worked in a blind fashion. All trainees attending the course had general experience in abdominal imaging. CTC images were reviewed by readers in $2 \mathrm{D}$ or 3D mode, or in combination, on the basis of their subjective preferences, using direct evaluation of native axial images and/or MPR reconstructions and/or virtual endoscopy and/or dissected views (Figs. 1-3).

Each reader was instructed to perform a systematic per-polyp analysis of each CTC dataset by classifying any detected polyp according to its size: small polyps $(<6 \mathrm{~mm})$, medium polyps (6-9 mm), large polyps (10-30 mm), and colonic masses (>30 mm). Each reader had $15 \mathrm{~min}$ time for assessing each dataset, after which results were compared with those provided by CAD software, which was thus used as second reader. In the 5 min allocated for the CAD sessions, CAD marks were analyzed by scrolling through the dataset. Raters operated in a blind fashion in both CAD-unassisted and CAD-assisted reading sessions. A diagram summarizing the procedural workflow of the study is provided in Fig. 4.

During the CAD-unassisted 15 -min reading session, readers recorded their results on a pre-printed form, on which they reported the slice number of the lesion, its maximum diameter, its segmental location, and its morphology. All suspected lesions had to be marked. In the CAD-assisted session, readers had to verify the correspondence between marked lesions and CAD-marked polyp candidates, but also reviewed CAD marks which had not eventually been marked at CAD-unassisted reading. The final decision was made by combining the polyp candidates selected in the CAD-unassisted and CAD-assisted reading settings.

As stated above, lesions were classified according to the CRADS criteria and the correspondence between CAD-unassisted and CAD-assisted marks was based on CT slice number and maximum diameter ( $1 \mathrm{~mm}$ mismatch between candidate measurement was tolerated). Anyway, all CAD software was designed to mark polyp candidates with a colored label, so that correspondence between them and those marked in the CAD-unassisted reading could easily be identified.

After the CAD-unassisted and CAD-assisted reading sessions, feedback was provided for each case; a tutor showed the same cases read by novices in a plenary session, and addressed the lesions con-

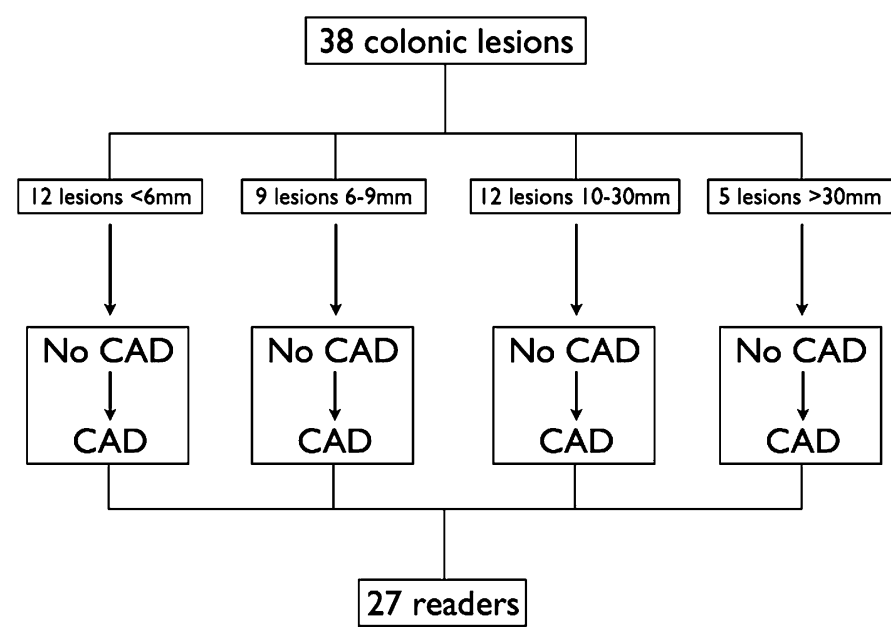

Fig. 4. Diagram illustrating criteria for case classification and analysis. 
Table 2

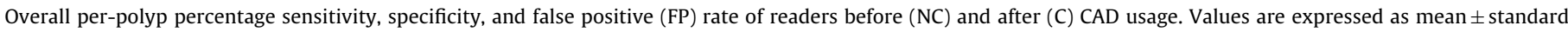
deviation.

\begin{tabular}{|c|c|c|c|c|c|c|c|c|c|}
\hline Lesion size & $\%$ sens $\mathrm{NC}$ & $\%$ sens C & $p$ & $\%$ spec NC & \% spec C & $p$ & FP rate NC & FP rate $C$ & $p$ \\
\hline$<6 \mathrm{~mm}$ & $18.52 \pm 16.56$ & $23.45 \pm 17.61$ & 0.0018 & $99.71 \pm 1.03$ & $99.29 \pm 1.86$ & 0.1855 & $0.0028 \pm 0.0103$ & $0.0071 \pm 0.0186$ & 0.1846 \\
\hline $6-9 \mathrm{~mm}$ & $28.70 \pm 10.16$ & $31.17 \pm 10.99$ & 0.0027 & $98.57 \pm 3.23$ & $98.57 \pm 3.23$ & 1 & $0.0142 \pm 0.0323$ & $0.0142 \pm 0.0323$ & 1 \\
\hline $10-30 \mathrm{~mm}$ & $53.08 \pm 21.20$ & $56.37 \pm 21.33$ & 0.0086 & $97.19 \pm 2.88$ & $96.93 \pm 2.92$ & 0.1618 & $0.0281 \pm 0.0288$ & $0.0307 \pm 0.0292$ & 0.1612 \\
\hline$>30 \mathrm{~mm}$ & $35.56 \pm 31.05$ & $35.56 \pm 31.05$ & 1 & $99.44 \pm 1.20$ & $99.44 \pm 1.20$ & 1 & $0.0056 \pm 0.0120$ & $0.0056 \pm 0.0120$ & 1 \\
\hline
\end{tabular}

firmed by endoscopy. During the 3-day course, with 20 min per case (26 cases), a total of $9 \mathrm{~h}$ of training was counted. Direct training on workstations was interleaved with short lectures and discussion on CTC practical issues, such as patient preparation, insufflation, and CTC indications. This format allowed, on one hand, to complete the training program with essential information for the clinical implementation of the technique, and on the other hand, to reduce readers' fatigue after assessing multiple cases (four cases were read in each session, interleaved with 1-2 lectures of 15-20 min each).

In the 5-h training session, readers were trained to starting a CAD session and informed about the pros and cons of the CAD; however, a dedicated lecture on CAD principles and performance was given by one expert in this field (DR).

\subsection{Statistical analysis}

For each rater and lesion size category, sensitivity and specificity before and after CAD usage were calculated and expressed as mean \pm standard deviation. As all CTC datasets contained at least one lesion, for each lesion size category true negative cases were computed as the sum of cases from the remaining categories minus false positive cases belonging to the category under examination.

In contrast with other works [9,13], in which reader response was coded in terms of a probability scale or score expressing the rater's subjective degree of confidence about the likelihood of a finding to be a lesion, we preferred to record each rater's response as a dichotomous variable $(0=$ no lesion; $1=$ lesion detected $)$, so to resemble as closely as possible the routine clinical scenario, in which the reporting radiologist is required to decide between lesion presence or absence. Given that every rater operated blinded and independently from each other, overall per-polyp sensitivity and specificity of raters as a group before and after CAD-assisted reading were compared by means of two-tailed paired Student's $t$ test.

In order to assess the potential impact of CAD on the performance of every single rater, sensitivity before and after CAD was also compared for each reader and lesion size by means of twotailed McNemar test.

Statistical analysis was carried out by using software (MedCalc version 10.3, www.medcalc.be; GraphPad Prism 5.0b, www.graphpad.com). A $p$-value less than 0.05 was considered statistically significant.

\section{Results}

Overall values of sensitivity, specificity, and false positive (FP) rate in the reader group ranked by lesion size before and after CADassisted reading, respectively, are reported in Table 2 .

Overall reader sensitivity was significantly higher after CADassisted reading than before for all lesion sizes, except for lesions larger than $30 \mathrm{~mm}$.

Neither overall specificity nor FP rate of readers was significantly different before and after CAD for any lesion size.

Diagnostic performance of each reader in terms of sensitivity and specificity for each lesion size category is illustrated in Graphs 1 and 2, respectively. Sensitivity of each single rater did not significantly differ before and after CAD for any lesion size category $(p>0.05)$.

\section{Discussion}

CAD is a useful tool for assisting the radiologist in the diagnosis of several pathological conditions, especially in an oncological setting. Its potential applications have extensively been investigated
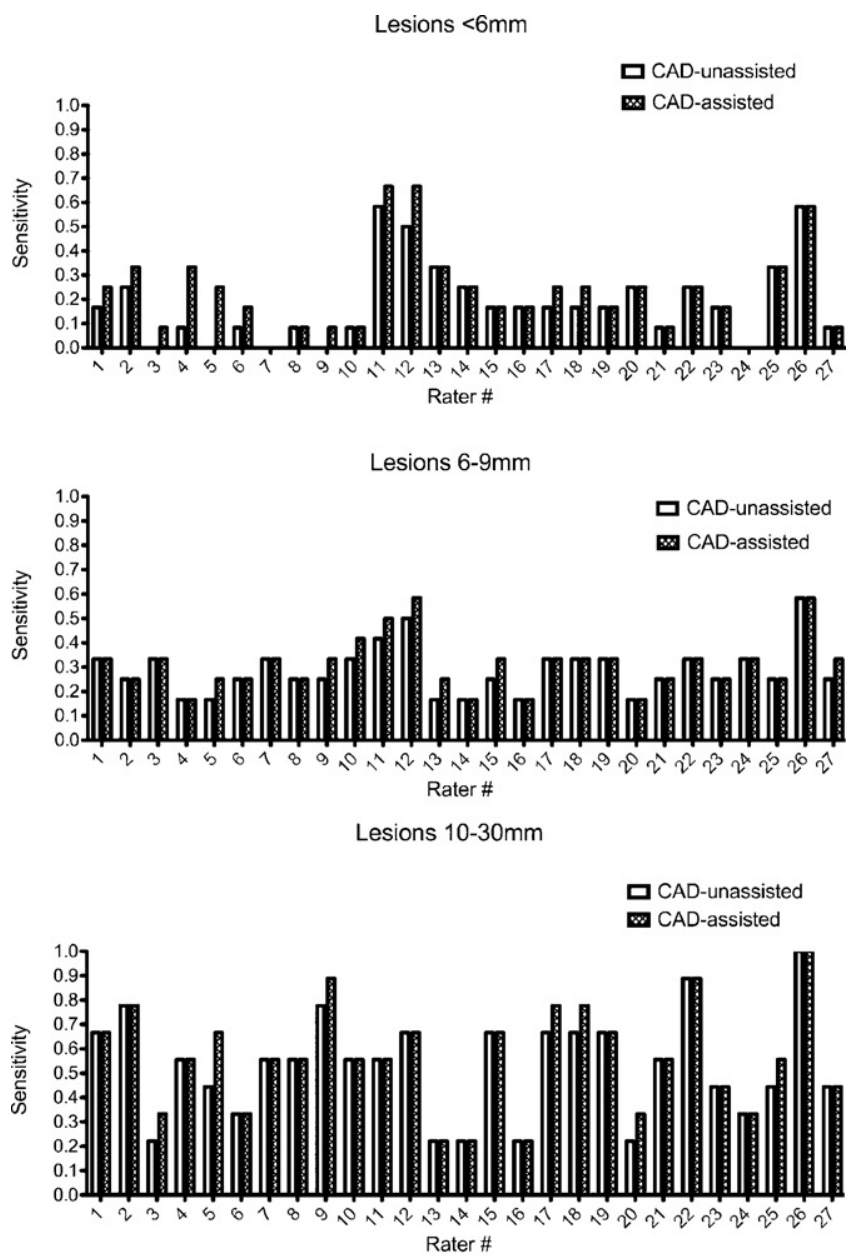

Lesions $>30 \mathrm{~mm}$

口 CAD-unassisted O CAD-assisted

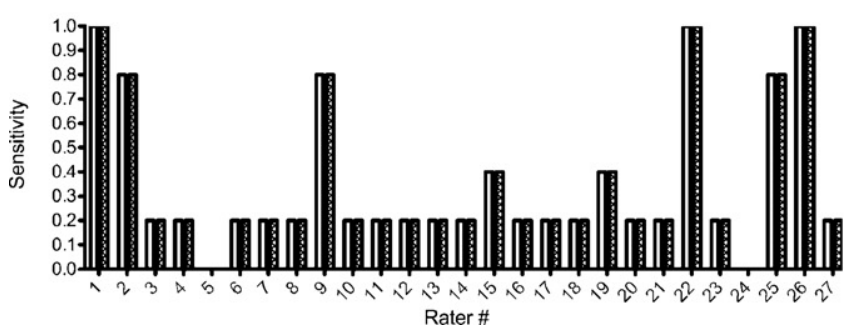

Graph 1. Sensitivity of each single rater before and after CAD usage according to lesion size: <6 mm (a), 6-9 mm (b), 10-30 mm (c), >30 mm (d). 
Lesions $<6 \mathrm{~mm}$

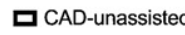
. $\mathrm{CAD}$-assisted
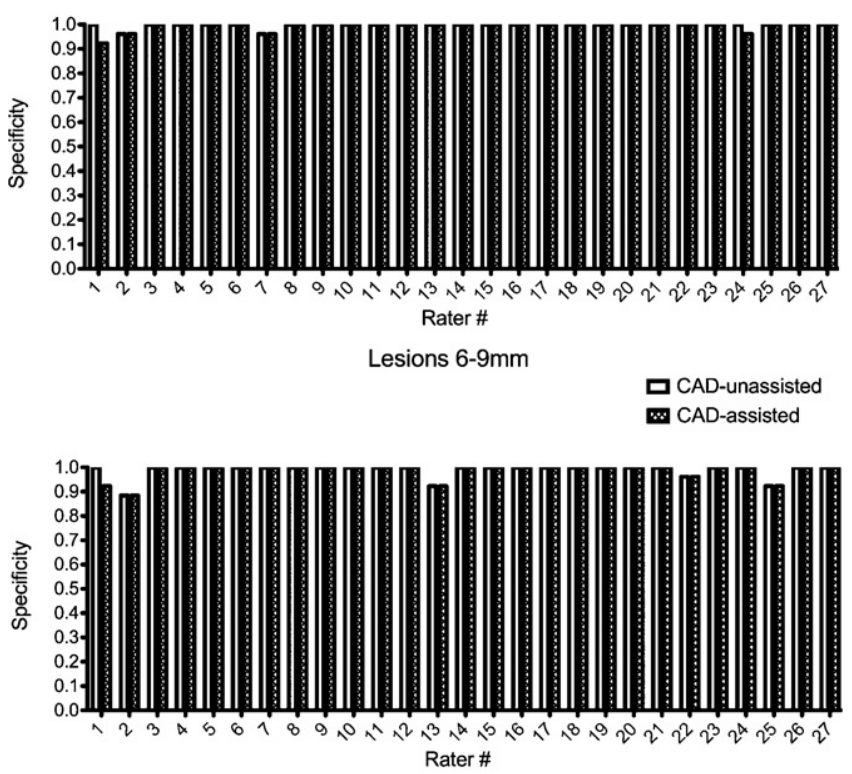

Lesions 10-30mm 口 CAD-unassisted . $\mathrm{CAD}$-assisted

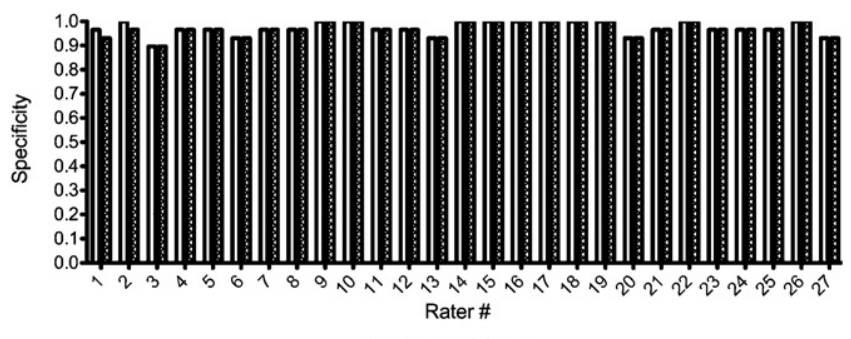

Lesions $>30 \mathrm{~mm}$

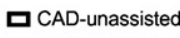

m CAD-assisted

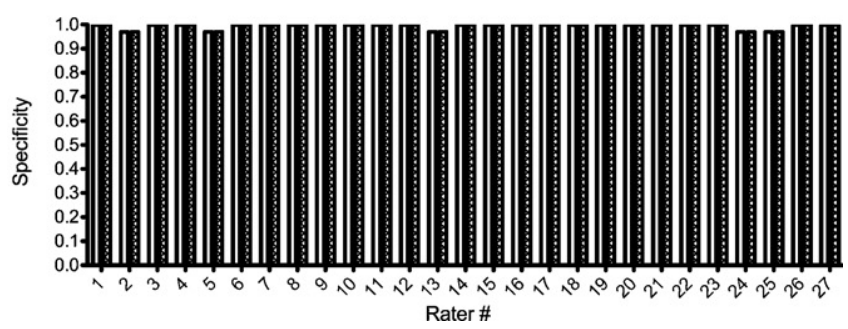

Graph 2. Specificity of each single rater before and after CAD usage according to lesion size: $<6 \mathrm{~mm}$ (a), 6-9 mm (b), 10-30 mm (c), >30 mm (d).

for the prediction of lung cancer in smokers, screening mammography, and CT colonography. In this latter field, attention has been paid to the effectiveness of CAD for assisting relatively inexperienced readers in the interpretation of diagnostic images.

Our results show that usage of CAD for lesion detection on CTC images can significantly improve the lesion detection rate of inexperienced readers on a rater population basis. In particular, CAD usage leads to a significant increase in the detection rate of lesions sized less than $30 \mathrm{~mm}$, while no difference exists between detection rates of colonic masses $(>30 \mathrm{~mm})$. These data are in line with those from other authors $[13,11]$, who report a significant increase in sensitivity when CAD is used by inexperienced investigators in a second reader paradigm. The lack of an increase for the detection of colonic masses can be explained by the fact that CAD software is not suited for detecting such large lesions, which are supposed to be easily recognizable by human readers alone. Despite this, it is interesting that reader sensitivity tends to increase with increasing lesion size up to $30 \mathrm{~mm}$, but falls in the case of colonic masses: this finding could be explained by the fact that inexperienced readers may actually miss such large lesions due to their basic inability to read CTC images and interpret colonic anatomy, which exposes them to the risk of gross image interpretation errors. On the other hand, the progressive increase in sensitivity with increasing lesion size up to $10 \mathrm{~mm}$ may be explained by the fact that inexperienced readers tend to overlook smaller lesions owing to perceptual errors, as suggested by Slater et al. [5]. The high variability in lesion sensitivity may confirm this hypothesis, suggesting that lesion detection in inexperienced readers may be substantially hampered by lack of skill in applying systematic diagnostic criteria for lesion seeking, although all readers have recently received an intensive dedicated training course.

Despite the statistically significant improvement in sensitivity brought by CAD, sensitivity value as intended in absolute terms is poor both before and after CAD usage. Other papers [5,9] confirm that CAD is of limited value when primary diagnosis is made by a reader without a previous substantial training in CTC. In particular, in the work by Slater et al. [5] sensitivity ranged from $7.1 \%$ to $28.6 \%, 16.7 \%$ to $41.7 \%$, and $16.7 \%$ to $83.3 \%$ for polyps sized $1-5 \mathrm{~mm}$, 6-9 $\mathrm{mm}$, and $\geq 10 \mathrm{~mm}$, respectively. On the other hand, Halligan et al. [9] have reported that on average, even with CAD, detection rate was $51 \%$ for polyps above $10 \mathrm{~mm}$ and $38.2 \%$ for polyps sized between 6 and $9 \mathrm{~mm}$.

It is noteworthy that, although sensitivity calculated on the whole rater population before and after CAD increases in a statistically significant manner for lesions below $30 \mathrm{~mm}$, sensitivity of individual raters does not show a significant difference before and after CAD for any of the raters involved in the study. However, we believe that, from an operative standpoint, individual reader sensitivity is more important than sensitivity of the whole rater population, as it expresses the effective ability of the CAD to improve a reader's diagnostic performance under real conditions.

Specificity tends to decrease, albeit not significantly and by a very little extent, after CAD application with lesions sized less than $6 \mathrm{~mm}$ and between 10 and $30 \mathrm{~mm}$, while it remains unchanged for lesions sized between 6 and $9 \mathrm{~mm}$, and above $30 \mathrm{~mm}$. Potential CAD-related loss of specificity is a known phenomenon that occurs also with experienced readers [12,14], due to the fact that CAD algorithms are primarily designed to detect lesions as alterations of the normal bowel wall by means of automated geometric criteria, while lesion characterization is an issue in which the radiologist's experience and judgment are more directly involved and, at least as of currently, much more difficult to be reproduced through dedicated algorithms.

Our findings raise the question of the effectiveness of CAD for improving diagnostic performance of inexperienced readers and focus the attention on the importance of adequate CTC training with the build-up of specialized skills. Hands-on courses are constantly offered by Academic Centers and Scientific Societies, as the most effective way to educate radiologists to the interpretation of CTC datasets; however, there is an emerging consensus that one of the most important factors affecting the diagnostic performance of CTC is dedicated training and experience of the radiologist who interprets the examination [15-17]. In particular, it has been suggested that competence in CTC image interpretation cannot be assumed even after directed training via a database of 50 cases [15,17].

Our study has a limitation in that, because of the teaching aim of the CTC hands-on course, we used CAD software by three different manufacturers for evaluation of CTC datasets instead of a single CAD application for all users; this may theoretically entail some 
variability in the performance of CAD tools, with a potential impact on the readers' diagnostic gain after CAD usage. However, all CAD plugins operated with their own standard settings in order to maximize diagnostic performance under the widest possible range of conditions, and the fact that sensitivity for lesion detection did not improve for any of the readers corroborates the hypothesis that this finding is independent from the features of individual CAD packages.

Another limitation is the relatively low number of cases (26 patients with a total of 38 colonic lesions) that might have prevented us from finding a statistically significant difference in the diagnostic performance of readers with and without CAD assistance. However, no more cases could be included due to the limited time available in a 3-day course for case revision, also considering that additional time was spent for lectures and critical case review.

In conclusion, our data show that usage of CAD software for CTC image evaluation by inexperienced readers, despite increasing diagnostic sensitivity on a rater population basis, does not lead to significantly higher sensitivity of individual raters. This finding may indicate that CAD tools are unable to compensate for lack of reader experience, thus stressing the importance of adequate training (presumably greater than that achievable through a CTC hands-on course) in order for radiologists to gain a satisfactory degree of diagnostic accuracy for the assessment of CTC datasets in a clinical setting.

\section{Conflict of interest}

None.

\section{References}

[1] Burling D, Halligan S, Atchley J, et al. CT colonography: interpretative performance in a non-academic environment. Clin Radiol 2007;62(5):424-9.
[2] Thomas S, Atchley J, Higginson A. Audit of the introduction of CT colonography for detection of colorectal carcinoma in a non-academic environment and its implications for the national bowel cancer screening programme. Clin Radiol 2009;64(2):142-7.

[3] Blachar A, Levy G, Graif M, Sosna J. Computed tomography colonography ("virtual colonoscopy") in Israel: results of the National CT Colonography Survey of the Israeli Association of Abdominal Imaging and the Israeli Radiological Association. Isr Med Assoc J 2008;10(10):707-12.

[4] Burling D, Moore A, Taylor S, La Porte S, Marshall M. Virtual colonoscopy training and accreditation: a national survey of radiologist experience and attitudes in the UK. Clin Radiol 2007;62(7):651-9.

[5] Slater A, Taylor SA, Tam E, et al. Reader error during CT colonography: causes and implications for training. Eur Radiol 2006;16(10):2275-83.

[6] Pickhardt PJ, Lee AD, Taylor AJ, et al. Primary 2D versus primary 3D polyp detection at screening CT colonography. AJR Am J Roentgenol 2007;189(6):1451-6.

[7] Yoshida H, Dachman AH. CAD techniques, challenges, and controversies in computed tomographic colonography. Abdom Imaging 2005;30(1):26-41.

[8] Taylor SA, Halligan S, Burling D, et al. Computer-assisted reader software versus expert reviewers for polyp detection on CT colonography. Am J Roentgenol 2006;186(3):696-702.

[9] Halligan S, Altman DG, Mallett S, et al. Computed tomographic colonography: assessment of radiologist performance with and without computer-aided detection. Gastroenterology 2006;131(6):1690-9.

[10] Taylor SA, Charman SC, Lefere P, et al. CT colonography: investigation of the optimum reader paradigm by using computer-aided detection software. Radiology 2008;246(2):463-71.

[11] Mang T, Peloschek P, Plank C, et al. Effect of computer-aided detection as a second reader in multidetector-row CT colonography. Eur Radiol 2007;17(10):2598-607.

[12] Petrick N, Haider M, Summers RM, et al. CT colonography with computeraided detection as a second reader: observer performance study. Radiology 2008;246(1):148-56.

[13] Baker ME, Bogoni L, Obuchowski NA, et al. Computer-aided detection of colorectal polyps: can it improve sensitivity of less-experienced readers? Radiology 2007;245(1):140-9.

[14] Morimoto T, Iinuma G, Shiraishi J, et al. Computer-aided detection in computed tomography colonography: current status and problems with detection of early colorectal cancer. Radiat Med 2008;26(5):261-9.

[15] Taylor SA, Halligan S, Burling D, et al. CT colonography: effect of experience and training on reader performance. Eur Radiol 2004;14(6):1025-33.

[16] Soto JA, Barish MA, Yee J. Reader training in CT colonography: how much is enough? Radiology 2005 Oct;237(1):26-7.

[17] European Society of Gastrointestinal and Abdominal Radiology CT Colonography Group Investigators. Effect of directed training on reader performance for CT colonography: multicenter study. Radiology 2007;242(1):152-61. 